\title{
Gearbox damage identification using Ensemble Empirical Decomposition method
}

\author{
Kamil Jonak ${ }^{1, *}$, and Arkadiusz Syta $^{1}$ \\ ${ }^{1}$ Lublin University of Technology Mechanical Engineering Faculty, Institute of Technological Systems of Information, \\ Nadbystrzycka 38 D 20-618 Lublin Poland
}

\begin{abstract}
In this article, we have conducted a comparative analysis of vibration signals from helicopter aircraft propulsion transmissions, registered on an industrial research stand. We compared acceleration vibrations in the case of gears without physical damage and gears with one tooth missing. Based on recorded signals, we determined the values of indicators based on the statistical properties of signals and compared them with each other. For a more exact comparison, the distribution of the tested signals to the empirical modes using the EEMD (Ensemble Empirical Mode Decomposition) method was performed. This allows to treat individual modes as components of a signal at specific frequencies, and also prevents mixing of modes in individual components, which may take place in the classic EMD analysis. It should be noted that individual modes may correspond to characteristic frequencies for the operation of the transmission. When comparing the values of the most frequently used indicators, modes/frequencies in which the damage was most visible were indicated.
\end{abstract}

\section{Introduction}

Monitoring the condition of main transmission of the helicopter drive is extremely important for safety and operational reasons. Non-invasive gear condition evaluation methods use vibroacoustic signals in the timefrequency domain, on the basis of which the characteristic values of discriminant $[1,2,3]$, which correspond to safe working ranges, are determined. The analysis of registered transmission vibration signals is not an easy task, due to the complexity and non-linearity of the drive transmission process, as well as the accompanying noise. Frequently, the signal is decomposed into experimental modes (Empirical Mode Decomposition - EMD), which correspond to the characteristic vibration frequencies and which can be treated as new multidimensional signals subjected to further analysis. In the paper [4], the authors, by means of decomposition into experimental modes, identified acceleration and machine learning signals, and the degree of damage to the gear tooth and various types of damage in the bearings. In the paper [5], the authors, using the extended EMD method (Ensemble Empirical Mode Decomposition - EEMD) in combination with machine learning methods, compared different fault detection algorithms in toothed wheels and obtained the best damage classification for the nearest neighbour method. In turn, in [6], the authors monitored the condition of rolling bearings based on vibroacoustic signals. Due to a proper analysis of EEMD and MSPCA, they were able to detect the moment of damage on many time scales. A good review of the application of the EMD method and its extensions can be found in $[7,8]$. Frequency detection methods are also used [9], as well as non-linear methods, where non-linear effects in the case of damage are assumed [10]. The purpose of this work is to compare statistical indicators that characterise the condition of the transmission and indication of the transmission with damage using the EEMD method. The article is organised as follows: a description of the test stand and registered signals, the methods, and the results obtained and a summary.

\section{Setup and signal recording}

In order to carry out the gearbox fault diagnosis an experimental stand has been made. The stand consisted of the tested gearbox connected and powered with electrical motor via speed multiplier gearbox and water brake (Fig. 2). Examined gearbox (PG-4 from PZL SW-4 helicopter [11]) consisted of the single stage transmission gears with angular teeth (pinion head with 10 teeth and driven wheel with 42 teeth). During the experiment gearbox worked in constant conditions with rotating speed set on $6196 \mathrm{rpm}$. Experimental data was recorded regularly from transmission start along to the occurrence of damage. After the damage of transmission occurred, gearbox was replaced with new one, and ran once again. Vibration signals were acquired by two $3 \mathrm{~d}$ sensors (a type of sensors: Bruel \& Kjaer 4321) with a sampling frequency of $40 \mathrm{kHz}$. The acceleration time series recorded in one direction are presented in figure Fig. 1.

${ }^{*}$ Corresponding author: k.jonak@pollub.pl 

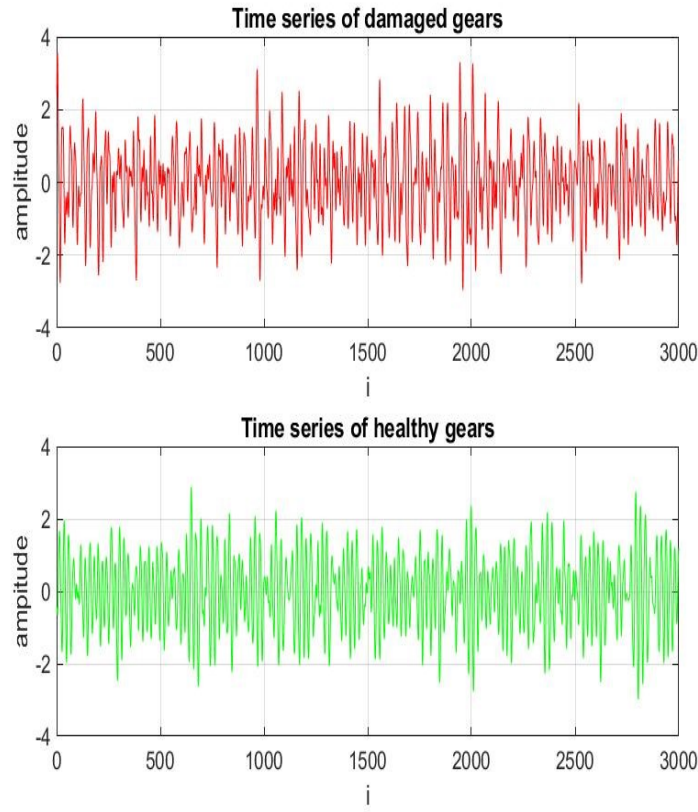

Fig. 1. The normalised amplitudes of the accelerations for both cases.

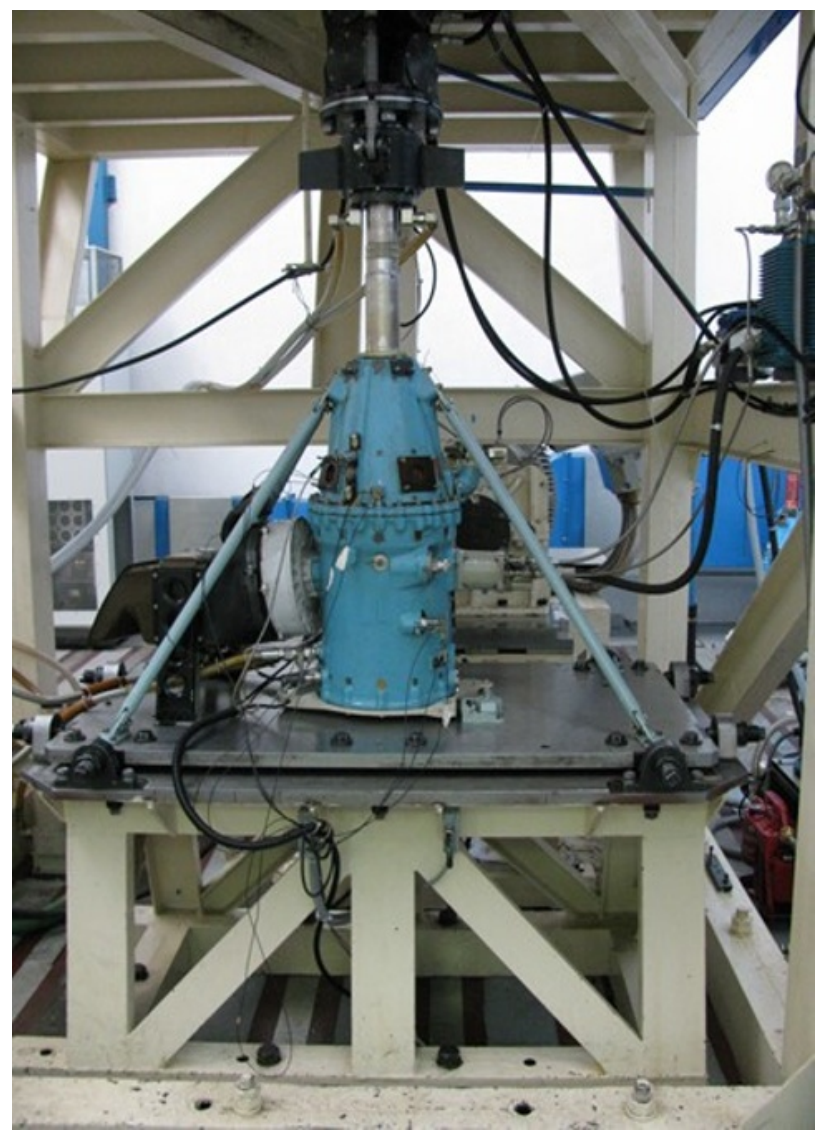

Fig. 2 Test stand with analysed gearbox.

For preliminary evaluation of the recorded signals, basic statistical measures, using ware/damage detection of rolling elements, were applied (Tab.1).
Table 1. Statistical measures. Where: $N$ is the number of samples; $i$ is the sample index; $x$ is the original signal; $\bar{x}$ is the mean $\mathrm{x} ; p k$ is the sample for the maximum positive peak of the signal; $x_{o-p k}$ is the value of $\mathrm{x}$ at $\mathrm{pk} ; r m s_{x}$ square root of the average of the sum of the squares of the signal samples; $d$ is the difference signal; $r$ is a residual signal; $M$ is a number of the current signal; $\bar{r}$-- mean of $\mathrm{r}$.

\begin{tabular}{|c|c|}
\hline $\begin{array}{c}\text { Method } \\
\text { Name }\end{array}$ & Definition \\
\hline Kurtosis & $K=\frac{N \sum_{i=1}^{n}\left(x_{i}-x\right)^{4}}{\left(\sum_{i=1}^{n}\left(x_{i}-x\right)^{2}\right)^{2}}$ \\
\hline Crest Factor & $C F=\frac{x_{o-p k}}{r m s_{x}}$ \\
\hline FM4 & $F M 4=\frac{N \sum_{i=1}^{n}\left(d_{i}-d\right)^{4}}{\left(\sum_{i=1}^{n}\left(d_{i}-d^{2}\right)^{2}\right.}$ \\
\hline NA4 & $N A 4=\frac{N \sum_{i=1}^{n}\left(r_{i M}-r_{M}\right)^{4}}{\frac{1}{M} \sum_{j=1}^{M}\left(\sum_{i=1}^{n}\left(r_{i j}-r_{j}\right)^{2}\right)^{2}}$ \\
\hline
\end{tabular}

We chose wear indicators based on the statistical properties of the registered series, often used in the literature[1]. The values of the indicators listed in Table 1. have been calculated for both time series and presented in Table 2.

Table 2. Values of the indicators.

\begin{tabular}{|c|c|c|}
\hline \multirow{2}{*}{$\begin{array}{c}\text { Method } \\
\text { Name }\end{array}$} & \multicolumn{2}{|c|}{ Values } \\
\cline { 2 - 3 } Kurtosis & Healthy & Damaged \\
\hline Crest Factor & 2.51 & 3.03 \\
\hline FM4 & 2.38 & 3.57 \\
\hline NA4 & 2.51 & 2.77 \\
\hline
\end{tabular}

Analysing the values of individual indicators, it can be seen that they are higher for the system with damage but are within the normal range. For example, kurtosis reaches a value of 3 for a standard Gaussian distribution that is characteristic of safe transmission operating conditions. In order to make a clearer distinction between the damaged system and without damage, we have decomposed the time series into experimental modes using the EEMD algorithm. 


\section{EEMD method}

The key function of empirical mode decomposition method (EMD) is a time-frequency signal analysis based on adaptive data decomposition into a series of IMFs [12]. These separated IMFs display signal characteristics which are contained in different frequency ranges. In recent years, the EMD algorithm was one of the most commonly used methods in terms of vibration signal analysis. However, despite its advantages, it also has some disadvantages. The major problem of the EMD algorithm is occurrence of intermittence that causes the mode mixing problem during IMF calculations. To overcome this problem $\mathrm{Wu}$ and Huang [13,14] proposed a new method called ensemble EMD (EEMD). The core of the EEMD algorithm is decomposition of the original signal with the addition of Gaussian noise into IMFs. Addition of different amplitude white noise differs the IMFs scales which suppress the modal mixing condition.

The EEMD algorithm goes as follows:

(1) Obtaining a new signal $z(t)$ by adding the white noise series $a(t)$ to the original signal $x(t)$ :

$$
z(t)=x(t)+a(t)
$$

(2) Decomposition of the $z(t)$ signal by EMD to obtain a set of IMFs:

$$
z(t)=\sum_{j=1}^{n} c_{j}(t)+r_{n}(t)
$$

(3) Repeat both previous steps $m$ times. Calculate $m$ groups of IMFs through addition of different amplitude of white noise $a_{i}(t), i=1 \sim m$ :

$$
\begin{aligned}
& z_{i}(t)=x(t)+a_{i}(t) \\
& z_{i}(t)=\sum_{j=1}^{n} c_{i j}(t)+r_{i j}(t)
\end{aligned}
$$

(4) Uphold the mean $c_{j}(t)(i=1,2, \ldots, m)$ of each $m$ IMFs as the final IMFs:

$$
c_{j}(t)=\frac{1}{m} \sum_{i=1}^{m} c_{i j}(t)
$$

The results of application of the EEMD algorithm into vibration signals from both gear boxes, health (a) and damaged (b), were shown in Fig. 3.

Treating individual fashions as separate signals corresponding to characteristic frequencies, we determined the values of indicators (Table 1) and compared them with each other.

The statements are presented in Figs. 3-6. When comparing the values of wear indicators, higher values of Kurtosis, Crest Factor and NA4 in the first mode are rewarded (Figures 3, 4, 6 show almost double increase, which does not appear in subsequent modes).

Differences in FM4 values are no longer so significant (Fig. 5) and are proportional to the values obtained for the output series (Table 2). It is worth noting that in subsequent modes the values of all indicators do not differ significantly from each other, both for gearbox without damage and with damaged teeth.

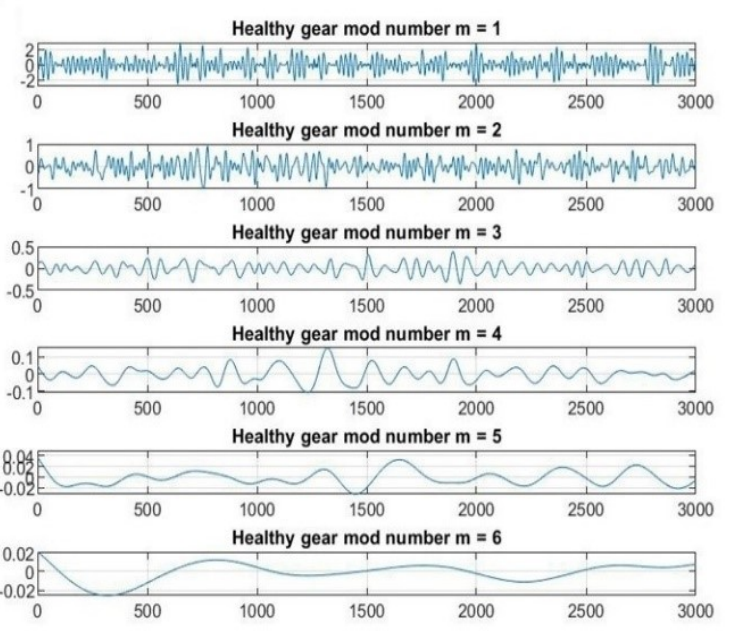

a)
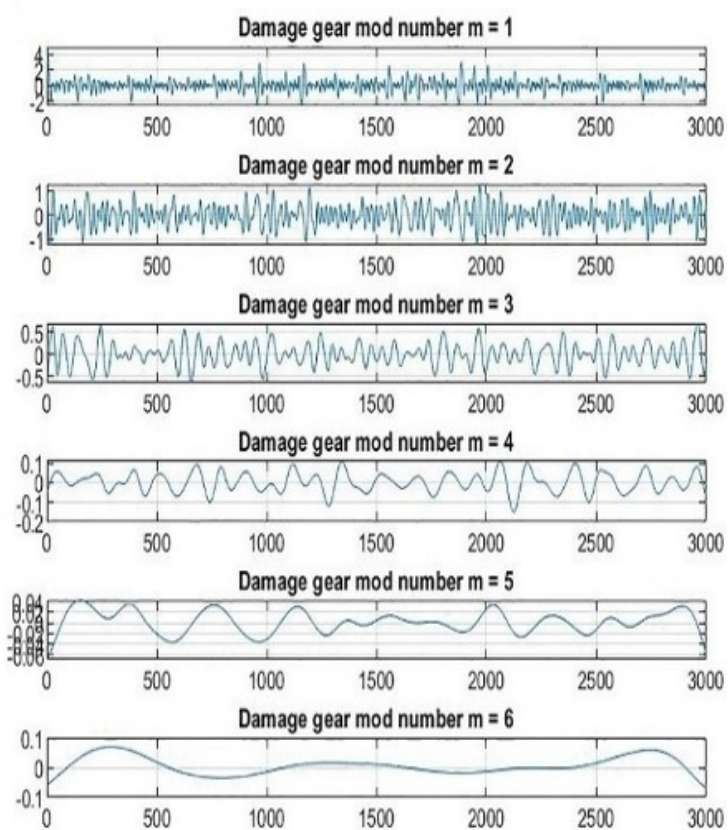

b)

Fig. 3. EEMD analysis results for healthy (a) and damaged (b) gearbox.

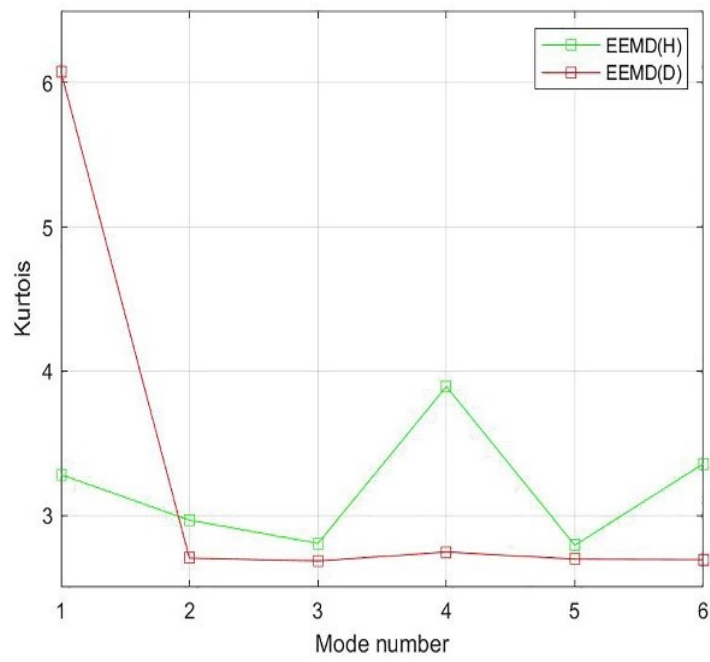

Fig. 4. Kurtosis calculated for the IMFs: healthy $(\mathrm{H})$ and damaged (D) gearbox. 


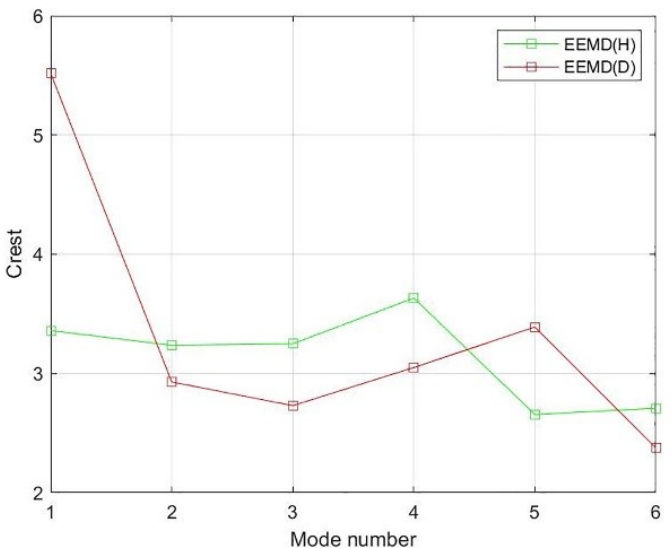

Fig. 5. Crest Factor calculated for the IMFs: healthy $(\mathrm{H})$ and damaged (D) gearbox.

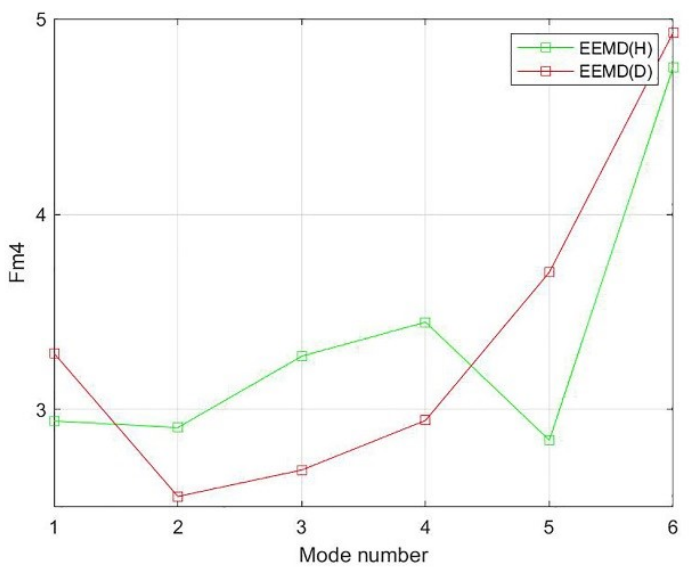

Fig. 6. FM4 calculated for the IMFs: healthy $(\mathrm{H})$ and damaged (D) gearbox.

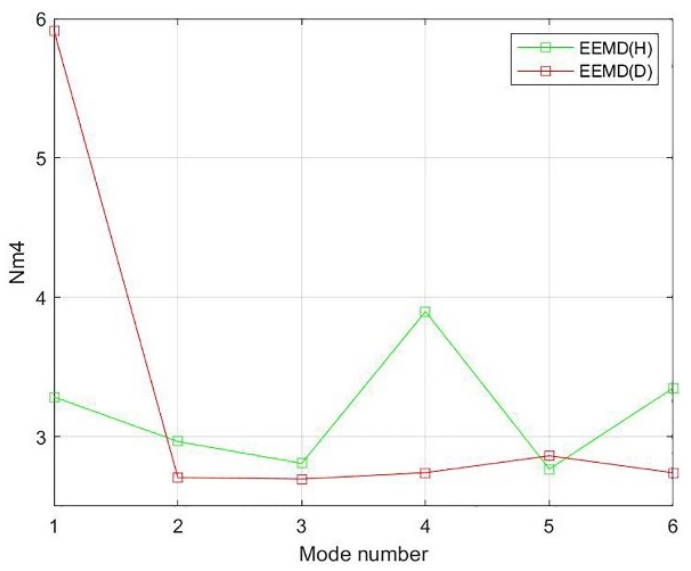

Fig. 7. NA4 calculated for the IMFs: healthy $(\mathrm{H})$ and damaged (D) gearbox.

\section{PCA analysis}

The new time series consist of modes of EEMD decompositions where analysed in scope of linear correlations and dimension reduction by the Principal Component Analysis (PCA) [10,12]. The PCA analysis can give an answer to question how much information/variability of the whole data is carried by a single mode. In other words, the PCA computes the eigenvectors of the covariance matrix and sorts corresponding eigenvalues in ascending order. Those eigenvalues reflect the amount of the variance explained by each of the principal components (the greater the variance of one variable, the greater the variability of the data is captured by this variable, please see Fig. 8).

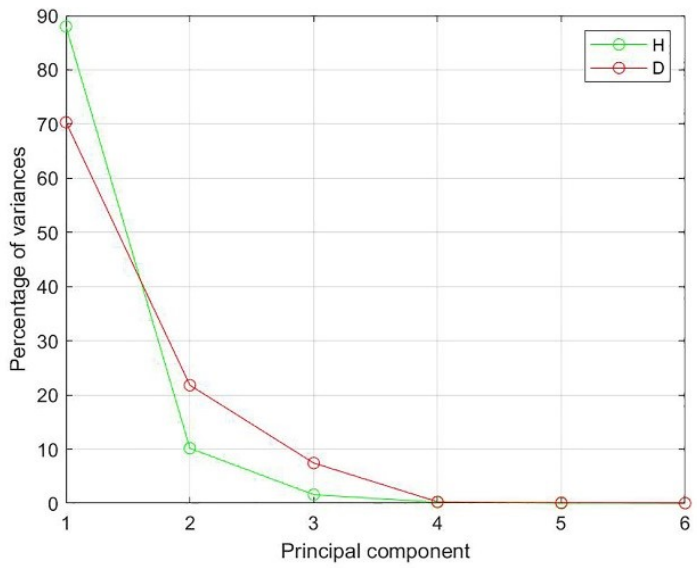

Fig. 8. The PCA results calculated for the modes: healthy $(\mathrm{H})$ and damaged (D) gearbox.

As one can notice, in both cases about $90 \%$ of the information contained in the data are retained by the first two principal components (for the healthy gear it is almost $100 \%$ ). It also means that first two modes are most significant and can be used for further analysis. We used the first two principal components to construct the project to two dimensions. On the other hand, one can approximately reconstruct data (a real coordinates) from the projection. In our case, we have information of all variables and we assume that projection error for reconstructed modes will be most significant in case of damaged gear. Such a comparison is visualised in figure Fig. 3, where the Root Mean Square of the reconstruction error is calculated for every mode.

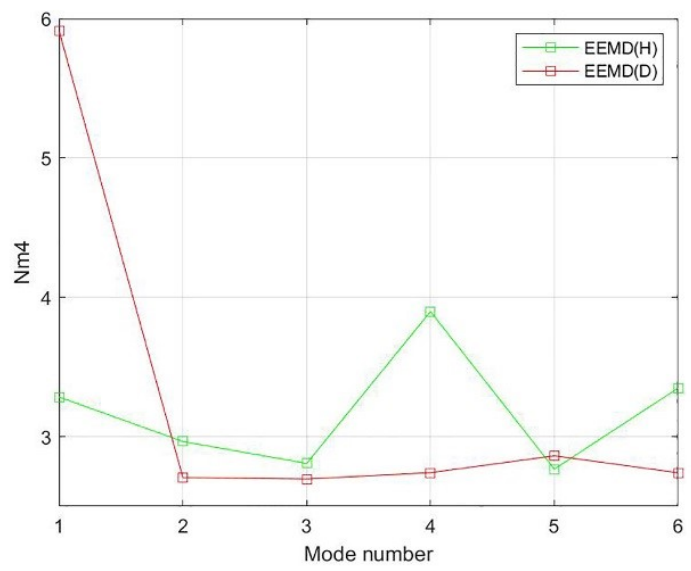

Fig. 9. The RMS of difference between EEMD modes and the reconstructed ones: healthy $(\mathrm{H})$ and damaged $(\mathrm{D})$ gearbox.

By analysing figure Fig. 9, significant differences can be seen between the reconstructed and original modes from 1 to 3 . The RMS of the reconstruction error is 
noticeable greater for the modes of the gear with damaged tooth (red) and reached the highest value of $20 \%$ for the second mode. The reconstruction error for the transmission signal without damage reaches the value of almost $10 \%$ also for the second mode. The analysis also shows that, in this case, modes from 4 to 6 can be omitted.

\section{Conclusions}

In this work we have made a comparative analysis of vibration signals recorded on the helicopter's main transmission. After dismantling one of the gears, it turned out that its teeth were damaged. Using statistical indicators calculated both for the original runs and their decomposition for experimental modes, we indicated the gear with the damaged tooth. It is worth paying attention to the values of discriminators based on statistical properties of time series. They all have a greater value for gears with damage, but close to 3 , which in the case of kurtosis corresponds to the normal distribution and corresponds to standard operating conditions. By decomposing the acceleration signals using the EEMD method, the distinction between the system with damage and without was more pronounced and values of the indicators increased almost twice for Kurtosis, Crest Factor and NA4. This means that in this case, thanks to the EEMD analysis, we achieved a clearer distinction between the efficient transmission and the deflected transmission. Additionally, using PCA analysis, we pointed out the modes in which the damage is most visible. However, to draw more general conclusions, more systematic experiments with various kinds of gear defects should be performed and statistically described.

\section{References}

1. V. Sharmaa, A. Pareya, Proc. Eng. 144 (2016)

2. H. Sohn, Ch. R Farrar, Smart Mater. Struct. 10 (2001)

3. A. Rai, S.H. Upadhyay, Trib Int. 96 (2016)

4. K.Vernekar, H. Kumar, K. V. Gangadharan, Sadhana 42 (2017)

5. C. Rajeswari, B. Sathiyabhama, S. Devendiran, K. Manivannan, JVE 17 (2015)

6. M. Zvokelj, S. Zupan, I. Prebil, Mech. Sys. Sig. Proc. 24 (2010)

7. Y. Lei, J. Lin, Z. He, M. J. Zuo, Mech. Sys. Sig. Proc. 35 (2013)

8. A. Rai,S.H.Upadhyay, Trib. Int. 96 (2016)

9. Ł. Jedliński, J. Jonak, Appl. S. Comp 30 (2015)

10. A. Syta, J. Jonak, Ł. Jedliński, G. Litak, J. Vib. Aco. 134, (2012)

11. J. Kisiel, J.Jonak, System monitorowania stanu przekładni śmigłowca SW-4 (Wydział IV Nauk Technicznych Polskiej Akademii Nauk, Uroczyste Posiedzenie Komitetu Budowy Maszyn, Wydział Mechaniczny Politechniki Lubelskiej, Lublin, 13.05. 2009)

12. Z. Wu, N. E. Huang, Adv. Adap. Da. Ana. 1 (2009)

13. Z. Wu, N. E. Huang, X. Chen, Adv. Adap. Da. Ana. 1 (2009)

14. Z. Wu, J. Feng, F. Qiao,Z-M.Tan, Phil. Trans. R. Soc. A 374 (2016) 